\title{
Near-band-edge slow luminescence in nominally undoped bulk ZnO
}

\author{
T. Monteiro, ${ }^{\text {a) }}$ A. J. Neves, M. C. Carmo, M. J. Soares, M. Peres, and J. Wang \\ Departamento de Física, Universidade de Aveiro, 3810-193 Aveiro, Portugal
}

E. Alves, E. Rita, and U. Wahl

Instituto Technológico e Nuclear, 2686-953 Sacavém, Lisboa, Portugal

(Received 10 December 2004; accepted 11 May 2005; published online 1 July 2005)

\begin{abstract}
We report the observation of slow emission bands overlapped with the near-band-edge steady-state luminescence of nominally undoped $\mathrm{ZnO}$ crystals. At low temperatures the time-resolved spectra are dominated by the emission of several high-energy bound exciton lines and the two-electron satellite spectral region. Furthermore, two donor-acceptor pair transitions at 3.22 and $3.238 \mathrm{eV}$ are clearly identified in temperature-dependent time-resolved spectroscopy. These donor-acceptor pairs involve a common shallow donor at $67 \mathrm{meV}$ and deep acceptor levels at 250 and $232 \mathrm{meV}$. (C) 2005 American Institute of Physics. [DOI: 10.1063/1.1946200]
\end{abstract}

\section{INTRODUCTION}

$\mathrm{ZnO}\left(E_{g} \sim 3.37 \mathrm{eV}\right.$ at room temperature) has received a great deal of attention due to its potential applications in optoelectronics and spintronics. Either nominally undoped or intentionally doped with $\mathrm{Al}, \mathrm{Ga}$, In, N, and/or rare-earth and transition-metal ions this semiconductor is a direct competitor with nitrides for potential electroluminescent devices and spintronic applications. Understanding the fundamental emission processes near the semiconductor band edge is of particular importance for applications of this oxide semiconductor in device emitters and light detectors for the ultraviolet and visible spectral region. ${ }^{1-9}$ At low temperatures the near-band-edge steady-state photoluminescence (PL) spectra of $\mathrm{ZnO}$ single crystals grown by seeded chemical-vapor transport (SCVT) is dominated by the donor bound exciton (BE) lines. ${ }^{1-7}$ The relative intensities of these BE lines is highly dependent on the growth process and the presence of impurities such as $\mathrm{H}, \mathrm{Al}, \mathrm{Ga}$, and $\mathrm{In}$, as well as postgrowth annealing treatments. ${ }^{1}$ On the low-energy side of the BE lines, the recombination processes have been attributed to two-electron satellite (TES) lines, LO-phonon replicas of BE's, donor-acceptor pair $\left(\mathrm{DA}_{x} \mathrm{P}\right)$ band at $3.22 \mathrm{eV}$, hereafter called DAP1, and the broad unstructured $2.5-\mathrm{eV}$ green emission band. The presence of structural defects has been associated with the $3.333-\mathrm{eV}$ line. ${ }^{1}$

In this paper we report on structural and optical characterization of nominally undoped bulk $\mathrm{ZnO}$ samples grown by SCVT. Besides the steady-state PL we present data obtained with time-resolved PL (TRPL) that allows for the identification of slow emission bands near the band edge of $\mathrm{ZnO}$, in the same spectral region where some of the BE's and TES lines occur. We show that these lines are observed overlapped with a slow violet band peaked near $3.0 \mathrm{eV}$. Moreover, TRPL also allows us to present a detailed analysis of other slow emission bands, such as DAP recombinations. Taking into account the measured temperature dependence of the steady-state and TRPL we propose models for the observed optical centers.

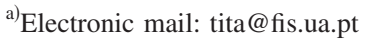

\section{EXPERIMENT}

High-quality $\mathrm{ZnO}$ single crystals grown by Eagle-Picher Technologies, LLC were analyzed. Crystalline quality was studied by Rutherford backscattering spectrometry and channeling (RBS/C). The measurements were performed using a 1-mm collimated $2-\mathrm{MeV}{ }^{4} \mathrm{He}^{+}$beam with the samples mounted in a computer-controlled two-axis goniometer with an accuracy of $0.01^{\circ}$. The backscattered particles were detected by two surface-barrier silicon detectors placed at $160^{\circ}$ and close to $180^{\circ}$ with respect to the beam direction and with energy resolutions of 13 and $16 \mathrm{keV}$, respectively. Particleinduced $\mathrm{x}$-ray emission (PIXE) was measured with a 10$\mathrm{mm}^{2} \mathrm{Si}(\mathrm{Li})$ detector of $150-\mathrm{keV}$ resolution and a $5-\mu \mathrm{m} \mathrm{Be}$ window. X-ray diffraction (XRD) was performed using a Philips instrument operating with $\mathrm{Cu} K \alpha$ radiation $(1.54178 \AA$ ) at $40 \mathrm{kV} / 50 \mathrm{~mA}$.

Steady-state PL was generated using the 325 -nm light from a cw $\mathrm{He}-\mathrm{Cd}$ laser, and an excitation power density less than $0.6 \mathrm{~W} \mathrm{~cm}^{-2}$. TRPL was carried out with a pulsed Xe lamp coupled to a monochromator and a boxcar system for detection (setup resolution from tens of microseconds to seconds). The samples were mounted in the cold finger of a closed-cycle helium cryostat and the sample temperature could be controlled in the range from $7 \mathrm{~K}$ up to room temperature (RT). The luminescence was measured using a Spex 1704 monochromator $\left(1 \mathrm{~m}, 1200 \mathrm{~mm}^{-1}\right)$ fitted with a cooled Hamamatsu R928 photomultiplier tube. The spectra have been corrected for the wavelength response of the optical system.

Raman spectra were taken at RT in a backscattering configuration with a Jobin Yvon Spex T64000 using the 514.5-nm line of an $\mathrm{Ar}^{+}$-ion laser as excitation source.

\section{RESULTS AND DISCUSSION}

Figure 1 shows the random and aligned RBS spectra for three axial directions of the wurtzite $\mathrm{ZnO}$ sample. The minimum yield along the $\langle 0001\rangle,\langle 11-23\rangle$, and $\langle 10-11\rangle$ axes is $2.4 \%, 5 \%$, and $8 \%$, respectively, which is an indication of the excellent crystalline quality of the single crystals. The 


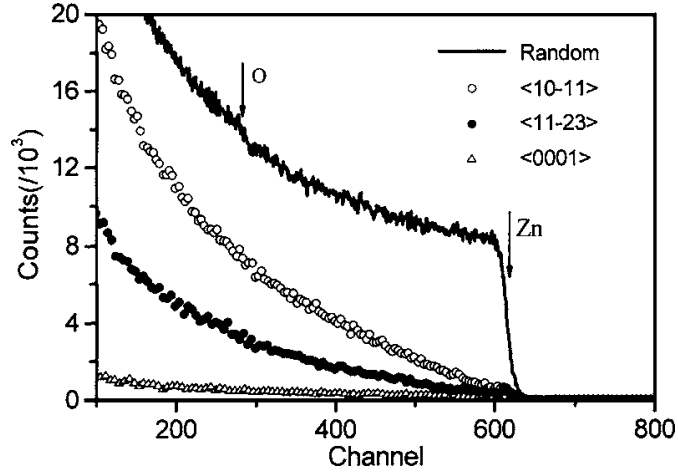

FIG. 1. Random and aligned spectra along the directions indicated for an undoped $\mathrm{ZnO}$ single crystal. The arrows indicate the position of the particles scattered at the surface by oxygen and zinc atoms.

smooth dechanneling rate over all the probed thickness (about $3 \mu \mathrm{m}$ for the $3-\mathrm{MeV}^{4} \mathrm{He}^{+}$), particularly for the tilt axes, reveals a nearly defect-free crystal over the entire depth. The crystalline quality of the samples was also confirmed by high-resolution x-ray diffraction (HRXRD). The $2 \theta$ scan shown in Fig. 2 shows a very strong $\mathrm{ZnO}$ (002) diffraction peak at $34.475^{\circ}\left(34.419^{\circ}\right.$ in $\mathrm{ZnO}$ powders $\left.{ }^{10}\right)$ with a full width at half maximum (FWHM) of $0.22^{\circ}$. The absence of diffraction peaks related to (100) and (101) planes indicates that the crystal quality is good and nearly strainfree.

A typical Raman spectrum of an as-grown $\mathrm{ZnO}$ wurtzite sample is shown in Fig. 3. The $E_{2}^{\text {high }}$ and $E_{2}^{\text {low }}$ vibration modes of wurtzite $\mathrm{ZnO}$ can be observed at 437.8 and $97.8 \mathrm{~cm}^{-1}$, respectively. Again, the peak position of the $E_{2}^{\text {high }}$ mode (almost the same as measured for $\mathrm{ZnO}$ powder ${ }^{11}$ ) and its FWHM of $7.2 \mathrm{~cm}^{-1}$ shows a nearly strain-free $\mathrm{ZnO}$ crystal. The peak at about $331 \mathrm{~cm}^{-1}$ is related to the phonon resonance at the edge of the Brillouin zone $(K-M-\Sigma) .{ }^{12}$ All these findings indicate that our $\mathrm{ZnO}$ samples have high crystalline quality.

The steady-state PL spectrum of the nominally undoped samples measured at $7 \mathrm{~K}$ shows a highly structured nearband-edge emission. Figure 4 shows the 3.1-3.37-eV energy region of the typical steady-state PL spectra of the samples used in this work as well as its temperature dependence. With above-band-gap excitation and low temperatures the most intense observed line in our samples is the $I_{4}$ transition. Lines $I_{8}, I_{5 / 6 / 6 a}, I_{9}$, and $I_{2}$ are also observed. ${ }^{1-7}$ At low temperatures the main transitions observed in this spectral region

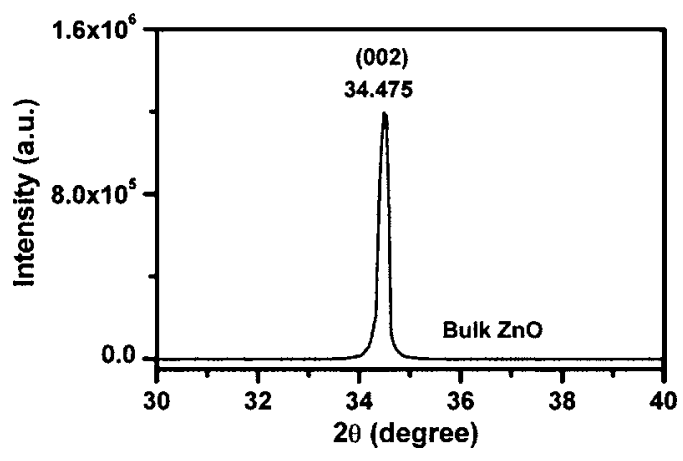

FIG. 2. X-ray-diffraction spectra of the studied sample.

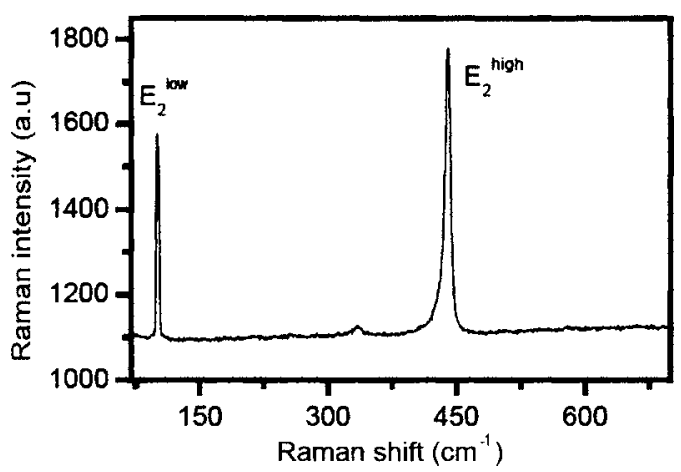

FIG. 3. RT Raman spectra of the studied sample obtained in backscattering configuration with a 514.5-nm $\mathrm{Ar}^{+}$laser line.

occur at $3.366 \mathrm{eV}\left(I_{2}\right), 3.365 \mathrm{eV}\left(I_{4}\right), 3.363 \mathrm{eV}\left(I_{5 / 6 / 6 a}\right)$, $3.362 \mathrm{eV}\left(I_{8}\right)$, and $3.359 \mathrm{eV}\left(I_{9}\right)$. On the low-energy side, lines at 3.333, 3.322, 3.311 (shoulder), 3.291, 3.26, and $3.328 \mathrm{eV}$ (shoulder) are observed. Besides these lines the typical 3.22-eV DAP1 and its 72-meV LO-phonon replicas are clearly resolved, as well as the broad unstructured green emission band $(2.5 \mathrm{eV})$ on the low-energy side. Similar spectra have been reported by other authors, ${ }^{1,2,5,6}$ showing that the same optically active defects are present in as-grown $\mathrm{ZnO}$ single crystals. With increasing temperature, the intensity of the 3.22-eV DAP1 drops. Simultaneously, the 3.311and $3.238-\mathrm{eV}$ lines as well as minor peaks $72 \mathrm{meV}$ apart can be clearly distinguished (in some of our samples these lines are well defined even at low temperatures). Other authors ${ }^{2,13}$ reported similar observations and focused their attention on the 3.238-eV transition. They have attributed this transition to an electron-acceptor recombination $\left(e, A^{0}\right)$ suggesting $E_{A}$ values of 195 and $208 \mathrm{meV}$, respectively, for the nitrogenrelated acceptor energy level. Further increase in temperature allows us to observe a decrease in intensity of the 3.311-eV line followed by the appearance of a shoulder on the high-energy side displaced by near $20 \mathrm{meV}$ at $120 \mathrm{~K}$, as shown in Fig. 5. Simultaneously the increase in temperature promotes the shrinkage of the band gap as well as a shift to low energies of the steady-state PL lines.

At RT the steady-state PL is dominated by transitions

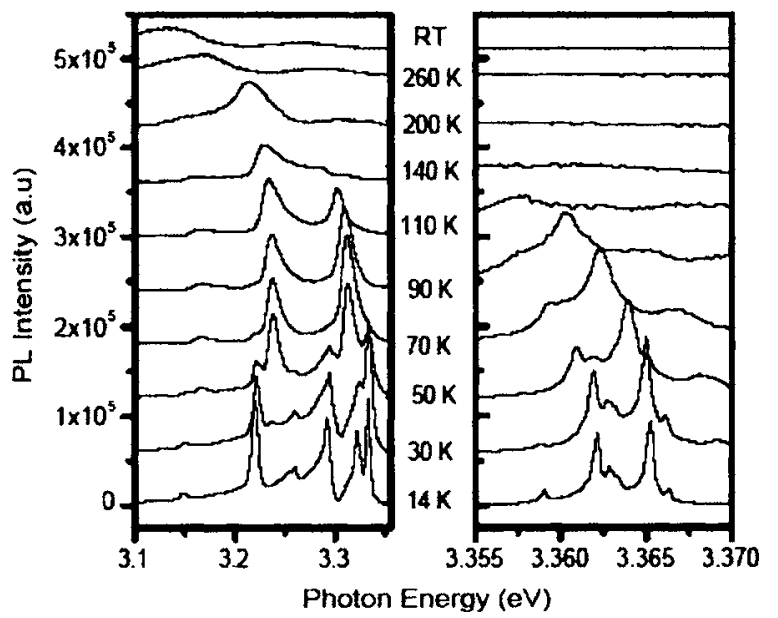

FIG. 4. Temperature dependence of the steady-state PL spectra of a nominally undoped $\mathrm{ZnO}$ sample. 


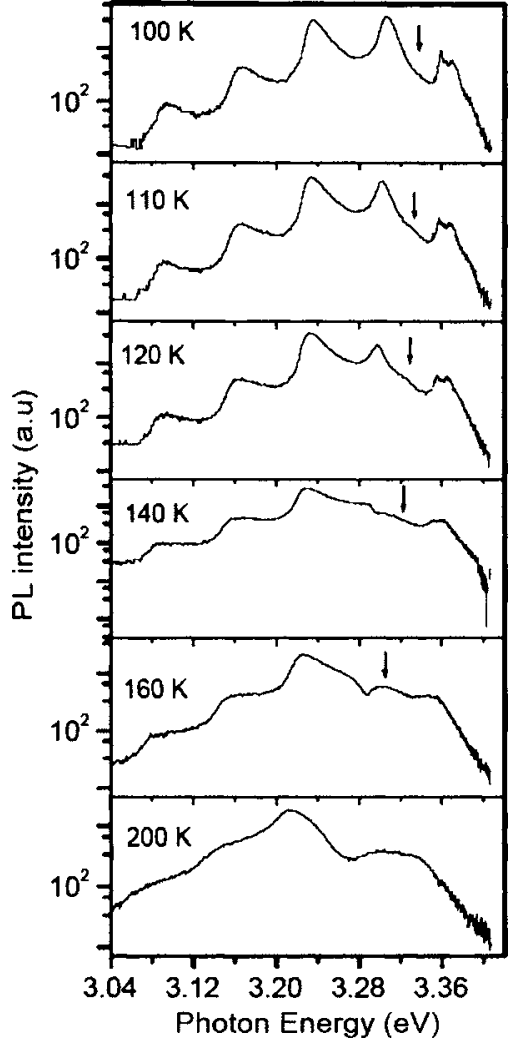

FIG. 5. Enlarged high-energy steady-state PL spectra and its temperature dependence for a nominally undoped $\mathrm{ZnO}$ sample.

peaked at ca. 3.3 and $3.14 \mathrm{eV}$ and their LO-phonon replicas as well as the green emission band at $2.5 \mathrm{eV}$.

Time-resolved spectroscopy permits a clearer understanding of the nature of some of these bands since this experimental technique is a useful tool to separate overlapping emissions with different lifetimes occurring in the same spectral region.

Several time-resolved spectra were measured at $14 \mathrm{~K}$, with above-band-gap excitation, and performed for a time delay $A$ (millisecond) and time window $B$ (millisecond) after the pulse lamp. Figure 6 shows the corresponding spectra taken for different $A$ and $B$ values together with steady-state PL for comparison. From these spectra it is clear that slow emission lines can be observed in the near band edge of

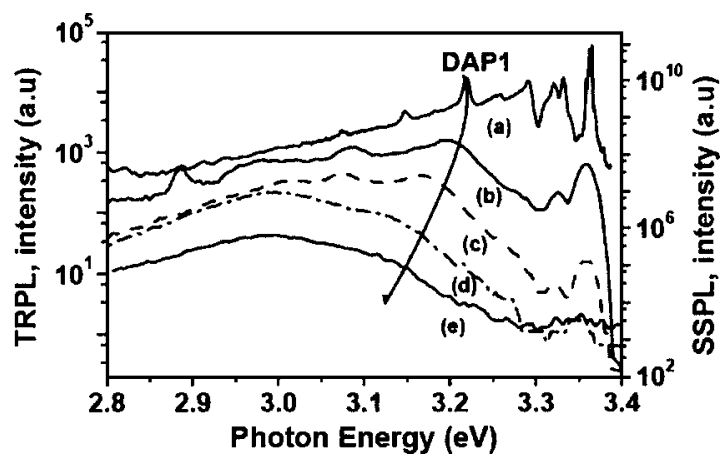

FIG. 6. (a) 14-K steady-state PL obtained with $\mathrm{He}-\mathrm{Cd}$ excitation. 14-K time-resolved spectra performed upon above-band-gap excitation for a time delay $A$ and time window $B$ (milliseconds) $(A / B)$ after the pulse lamp: (b) 0.05/0.02, (c) 0.08/0.05, (d) 5/10, and (e) 100/100.

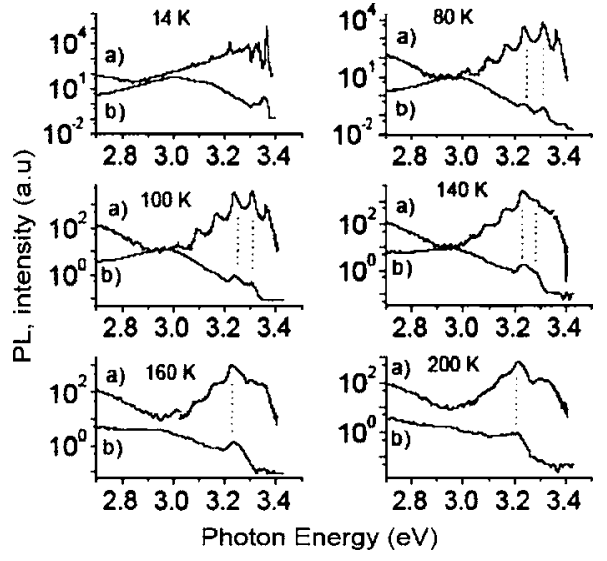

FIG. 7. Comparison between (a) steady-state PL upon He-Cd excitation and (b) time-resolved spectra performed with above-band-gap excitation for a time delay of $0.1 \mathrm{~ms}$ and time window of $15 \mathrm{~ms}$ after the lamp pulse for different temperatures.

nominally undoped bulk $\mathrm{ZnO}$. The faster emission bands correspond to the 3.36-, 3.328-, and 3.311-eV lines, currently attributed to the BE's and TES spectral emission. On the low-energy side of these lines the 3.22-eV DAP1 is overlapped with a slow emitting center at $3.0 \mathrm{eV}$ (violet band). In order to identify the different slow emitting centers we have performed time-resolved studies with different time delays and time windows. Reducing the intensity of the $3.0-\mathrm{eV}$ slow emission band using shorter delay times and time windows, the high-energy lines and the 3.22-eV DAP1 and its LOphonon replicas become clearly identifiable. The 3.22-eV emission shows a clear redshift with increasing delay times and time windows, as expected for a DAP recombination. For larger delays and time windows, most of the slow recombination processes merge in the wide violet band peaked near $3.0 \mathrm{eV}$ at $14 \mathrm{~K}$. Figure 7 shows the temperature dependence of the time-resolved PL with long delays $(0.1 \mathrm{~ms})$ and time windows $(15 \mathrm{~ms})$ for which the slower emitting centers are dominating. As previously mentioned (Fig. 4) the intensity of the steady-state 3.22-eV DAP1 PL quenches with increasing temperatures and is hardly observed for temperatures above 40 K. Comparing the time-resolved PL (Fig. 7) and steadystate luminescence (Fig. 4) is clear that the broad violet emission is still present even at high temperatures ( $T$ $>40 \mathrm{~K}$ ), becoming practically undetectable for temperatures above $160 \mathrm{~K}$ (see Figs. 7 and 8). This shows that the violet emission is definitely not correlated with the 3.22-eV DAP1 emission. A small Huang-Rhys factor has been observed for most of DAP transitions in II-VI compounds ${ }^{1,14}$ [for instance, the nitrogen-related 3.235-eV $\mathrm{DA}_{\mathrm{N}} \mathrm{P}$ observed in $\mathrm{ZnO}$ films intentionally doped with $\mathrm{N}$ has a Huang-Rhys of 0.8 (Ref. 14)]. Assuming a similar Huang-Rhys factor for the 3.22-eV DAP1 transition above mentioned it does not seem possible to assign the broad $3.0-\mathrm{eV}$ band to distant pair recombination.

Another interesting feature can be observed from Fig. 8 where a comparison of the steady-state and the time-resolved PL in a larger spectral scale is shown. As previously mentioned under steady-state PL conditions a broad unstructured green $2.5-\mathrm{eV}$ emission band is observed. Our time-resolved 


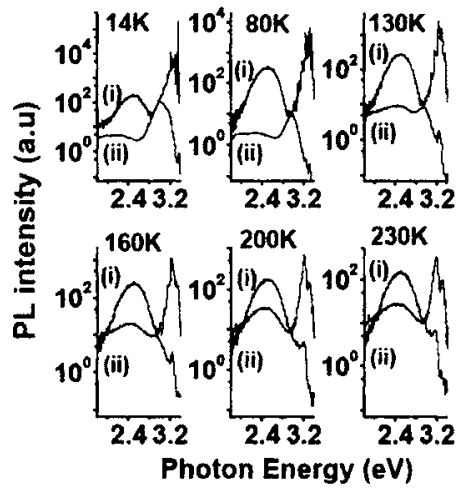

(a)

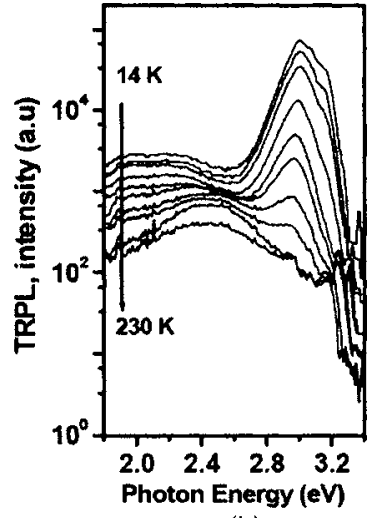

(b)
FIG. 8. (a) Large spectral region comparison between (i) steady-state PL upon $\mathrm{He}-\mathrm{Cd}$ excitation and (ii) time-resolved spectra performed upon above-band-gap excitation for a time delay of $0.1 \mathrm{~ms}$ and time window of $15 \mathrm{~ms}$ after the pulse lamp for different temperatures. (b) Temperature dependence of the time-resolved spectra performed as described in (a).

measurements in that spectral region show an overlap of the violet $(3.0 \mathrm{eV})$ and the green $(2.5 \mathrm{eV})$ emission band. After the quenching of the violet emission, the green band is well resolved and this emission band becomes the main slow luminescence observed at higher temperatures.

Recent reports attributed the green emission band to an intradefect triplet-singlet transition due to oxygen vacancies in $\mathrm{ZnO} .{ }^{1,15}$ However, up to now, there is no consensus in the literature concerning the nature of the green emission band: several intrinsic defects such as $V_{\mathrm{O}}, V_{\mathrm{Zn}}, \mathrm{Zn}_{i}$, and $O_{\mathrm{Zn}}$ have been proposed as well as extrinsic related defects such as $\mathrm{Cu}^{16-28}$ Despite the nature of the involved defect producing the $2.5-\mathrm{eV}$ emission it is clear that if a spin-forbidden tripletsinglet transition is involved in the recombination mechanism (as expected for the oxygen vacancy) a slow luminescence should be observed, in good agreement with our results.

Using shorter delay times $(20 \mu \mathrm{s})$, for which the faster emitting centers dominate, the intensity of the most energetic transitions increases. This suggests that they have shorter lifetimes. Under these conditions Fig. 9 shows a comparison between the steady-state and time-resolved PL for several temperatures. With increasing temperatures the lines at 3.36, 3.311, and $3.238 \mathrm{eV}$ and its LO-phonon replicas have lifetimes in the microsecond time scale or shorter. The emission at $3.36 \mathrm{eV}$ is shown to be faster than at 3.311 and $3.238 \mathrm{eV}$ since it cannot be detected at all for delay times above $100 \mu$ s while the other lines are still observed. At low temperatures the energy difference between the 3.311-, 3.238-, 3.166, and 3.094-eV lines are very similar, $\sim 72 \mathrm{meV}$, but with increasing temperatures the relative intensities and the energy difference between the 3.311-, and 3.238-eV lines do not remain constant. This result has been previously reported in cathodoluminescence by other authors. ${ }^{9}$ This behavior for the 3.238-, 3.166-, and 3.094-eV lines up to $200 \mathrm{~K}$ suggests that these lines can be assigned to vibronic transitions occurring at the same center. We also point out that the timeresolved spectra of the 3.238-eV line and its LO-phonon replicas are always slightly shifted to lower energies than the steady-state PL. This shows that the 3.238-eV emission can

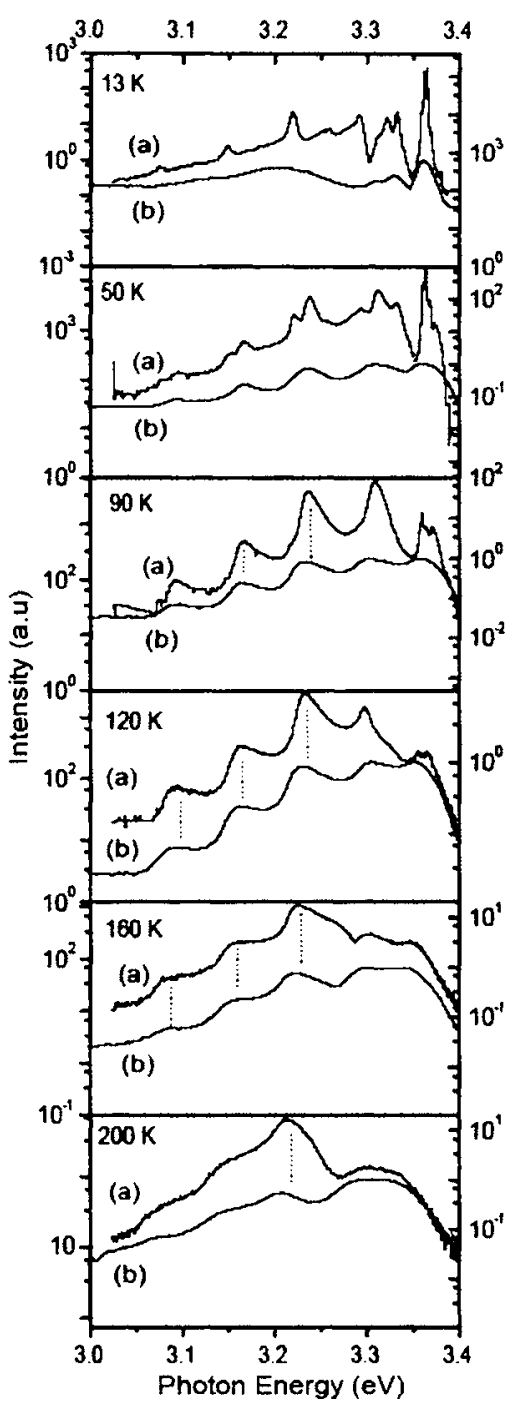

FIG. 9. Comparison between (a) steady-state PL upon He-Cd excitation and (b) time-resolved spectra performed with above-band-gap excitation for a time delay of $0.02 \mathrm{~ms}$ and time window of $0.05 \mathrm{~ms}$ after the lamp pulse for different temperatures.

be attributed to a DAP recombination (DAP2). The DAP nature of this emission is further reinforced by the fact that the $(3.238 \mathrm{eV})$ peak position does not follow the shrinkage of the band gap, as can be seen in Fig. 10. This is clearly not

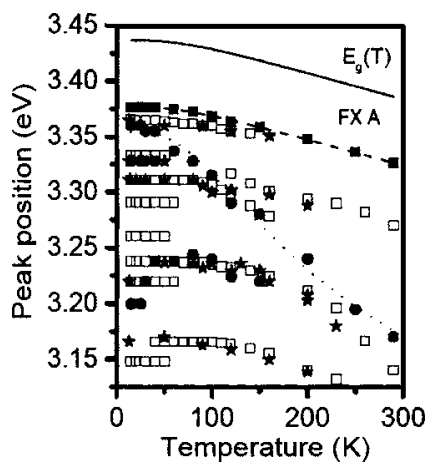

FIG. 10. Some peak position and their temperature dependence: open squares, steady-state photoluminescence lines; stars, time-resolved photoluminescence lines; full circles, obtained by photoluminescence excitation monitored in the band maximum of green emission $(2.5 \mathrm{eV})$. 
the expected behavior for an $\left(e, A^{0}\right)$ recombination as suggested by other authors. ${ }^{2,13}$

Following the observed data we believe that in our samples two DAP transitions are present even at low temperatures. The DAP transitions have zero-phonon lines at $3.22 \mathrm{eV}$ (DAP1) and $3.238 \mathrm{eV}$ (DAP2) and must involve a common shallow donor.

In intentionally doped and annealed samples where the 3.0-eV band is nearly absent we have been able to measure by time-resolved spectroscopy the distant pair recombination for DAP1 at $3.12 \mathrm{eV} .^{29}$ The transition energy of distant DAP recombination is given by ${ }^{30}$

$$
E(h \nu)=E_{g}-\left(E_{A}+E_{D}\right)+\frac{e^{2}}{4 \pi \varepsilon r},
$$

where $E_{g}$ is the band gap, $E_{A}$ and $E_{D}$ the acceptor and donor ionization energies respectively, $E_{C}=e^{2} / 4 \pi \varepsilon r$ represents the Coulomb interaction between the pair, $\varepsilon$ is the dielectric constant, and $r$ is the pair separation. Assuming infinite pair separation, we obtained $E_{A}+E_{D} \sim 317 \mathrm{meV}$ and an estimated average Coulomb energy of $100 \mathrm{meV}$, giving an average pair separation $\langle r\rangle$ of $17 \AA$.

Deep-level transient spectroscopy (DLTS) and admittance spectroscopy ${ }^{31}$ in similar crystals have found shallow electron traps at 37 and $67 \mathrm{meV}$ with concentrations of the order of $\sim 10^{16} \mathrm{~cm}^{-3}$. Assuming that the $67-\mathrm{meV}$ shallow donor is involved in both the 3.22-eV DAP1 and the 3.238 $-\mathrm{eV}$ DAP2 the acceptor levels must be located at 250 and $232 \mathrm{meV}$, respectively. Nitrogen has been associated with the acceptor involved in the DAP1 recombination. ${ }^{2,32}$ Two distinct nitrogen-related acceptors $\left(\mathrm{N}^{0}\right.$ and $\left.\mathrm{N}_{2}^{-}\right)$were observed using electron paramagnetic resonance (EPR). ${ }^{32}$ These authors associated the single shallower level with DAP1 and assumed a deeper level for the molecular nitrogen. After illumination and warming the crystals up to $100 \mathrm{~K}$ Garces et $\mathrm{al}^{32}$ also found a decrease of neutral nitrogen forms $\left(\mathrm{N}^{0}, \mathrm{~N}_{2}^{0}\right)$ and a simultaneous increase of the single ionized charge states $\left(\mathrm{N}^{-}, \mathrm{N}_{2}^{-}\right)$. In our samples, in the same temperature region where $\mathrm{N}^{0}$ is converted to $\mathrm{N}^{-}$and $\mathrm{N}_{2}^{0}$ is converted to $\mathrm{N}_{2}^{-}$, we observed the quenching of the photoluminescence intensity of the 3.22-eV DAP1 and the increase in intensity of the $3.238-\mathrm{eV}$ DAP2. If $\mathrm{N}^{0}$ is the acceptor involved in the 3.22-eV DAP1 then its energy level must be deeper than the 195 and $208 \mathrm{meV}$ calculated by Thonke et al. ${ }^{2}$ and Wang et al. ${ }^{13}$ From our TRPL data the level should be located at $250 \mathrm{meV}$ above the valence band. If we assume that the molecular nitrogen form is involved in the 3.238-eV DAP2 then our data suggest that the level should be shallower than the $\mathrm{N}^{0}(232 \mathrm{meV}$ above the valence band). However, despite the fact that nitrogen is involved on both DAP recombination processes we cannot rule out that the shallowest nitrogen level could be related to other nitrogen forms or complex defects.

The presence of these two DAP transitions gives rise to a continuum distribution of electronic states well within the band gap. When photoluminescence excitation is monitored on the maximum of the green band it is clear that besides the onset absorption at $3.05 \mathrm{eV}$ an indirect population via electronic states that are resonant with these two DAP transitions occurs, ${ }^{33}$ as can be observed from Fig. 10. These data are further indication that the slow emitting character observed in the green band could be due to this indirect mechanism rather than a prompt recombination.

\section{CONCLUSIONS}

Using time-resolved spectroscopy measurements we were able to prove that in high-quality nonintentionally doped $\mathrm{ZnO}$ bulk samples the typical near-band-edge steadystate PL spectra are due to an overlap of slow emitting centers. The data reveal that most of steady-state recombination processes are overlapped with a broad violet band peaked near $3.0 \mathrm{eV}$ that could be observed up to $160 \mathrm{~K}$. At RT the main slow emitting center corresponds to the green band $(2.5 \mathrm{eV})$ that is likely to be indirectly populated.

Our data are consistent with the presence, even at low temperatures, of two nitrogen-related DAP transitions at $3.22 \mathrm{eV}$ (DAP1) and at $3.238 \mathrm{eV}$ (DAP2), involving a common $67-\mathrm{meV}$ shallow donor level and acceptor levels at 250 and $232 \mathrm{meV}$, respectively.

\section{ACKNOWLEDGMENTS}

The authors would like to acknowledge the financial support by Fundação para a Ciência e Tecnologia/FEDER (POCTI/CTM/45236/02). Four of the authors (M.P., J.W., E.R., and U.W.) would like to thank the Fundação para a Ciência e Tecnologia for their grants. The authors gratefully acknowledge to Dr. R. S. Ferreira for her help in the experiments.

${ }^{1}$ B. K. Meyer et al., Phys. Status Solidi B 241, 231 (2004), and references therein.

${ }^{2}$ K. Thonke, Th. Gruber, N. Teofilov, R. Schonfelder, A. Waag, and R. Sauer, Physica B 308, 945 (2001).

${ }^{3}$ D. C. Reynolds, D. C. Look, B. Jogai, C. W. Litton, T. C. Collins, W. Harsch, and G. Cantwell, Phys. Rev. B 57, 12151 (1998).

${ }^{4}$ C. Boemare, T. Monteiro, M. J. Soares, J. G. Guilherme, and E. Alves, Physica B 308-310, 985 (2001)

${ }^{5}$ D. W. Hamby, D. A. Lucca, M. J. Klopfstein, and G. Cantwell, J. Appl. Phys. 93, 3214 (2003)

${ }^{6}$ L. Wang and N. C. Gilles, J. Appl. Phys. 94, 973 (2003).

${ }^{7}$ Y. M. Strzhemechny et al., Appl. Phys. Lett. 84, 2545 (2004).

${ }^{8}$ A. A. Toropov et al., Phys. Rev. B 69, 165205 (2004).

${ }^{9}$ N. Ohashi, T. Sekiguchi, K. Aoyama, T. Ohgaki, Y. Terada, I. Sakaguchi, T. Tsurumi, and H. Haneda, J. Appl. Phys. 91, 3658 (2002).

${ }^{10}$ R. B. Heller, J. McGannon, and A. H. Weber, J. Appl. Phys. 21, 1283 (1950).

${ }^{11}$ X. Wang, S. Yang, J. Wang, M. Li, X. Jiang, G. Du, X. Liu, and R. P. H. Chang, J. Cryst. Growth 226, 123 (2001).

${ }^{12}$ J. M. Calleja and M. Cardona, Phys. Rev. B 16, 3753 (1977).

${ }^{13}$ L. Wang and N. C. Gilles, Appl. Phys. Lett. 84, 3049 (2004).

${ }^{14}$ A. Zeuner, H. Alves, D. M. Hofmann, B. K. Meyer, A. Hofmann, U. Haboeck, M. Strassburg, and M. Dworzak, Phys. Status Solidi B 234, R7 (2002).

${ }^{15}$ F. Leiter, H. Alves, D. Pfisterer, N. G. Romanov, D. M. Hofmann, and B. K. Meyer, Physica B 340-342, 201 (2003).

${ }^{16}$ S. Takata, T. Minami, H. Nanto, and T. Kawamura, Phys. Status Solidi A 65, k83 (1981).

${ }^{17}$ K. Vanheusden, W. L. Warren, C. H. Seager, D. R. Tallant, J. A. Voigt, and B. E. Gnade, J. Appl. Phys. 79, 7983 (1996).

${ }^{18}$ B. Lin, Z. Fu, Y. Jia, and G. Liao, J. Electrochem. Soc. 148, G110 (2001).

${ }^{19}$ S. A. Studenikin, N. Golego, and M. Cocivera, J. Appl. Phys. 84, 2287 (1998).

${ }^{20}$ A. van Dijken, E. A. Meulenkamp, D. Vanmaekelbergh, and A. Meijerink, J. Lumin. 90, 123 (2000). 
${ }^{21}$ A. F. Kohan, G. Ceder, D. Morgan, and C. G. Van de Walle, Phys. Rev. B 61, 15019 (2000).

${ }^{22}$ S. B. Zhang, S. H. Wei, and A. Zunger, Phys. Rev. B 63, 075205 (2001).

${ }^{23}$ R. Dingle, Phys. Rev. Lett. 23, 579 (1969).

${ }^{24}$ I. Broser and R. K. F. Germer, Solid-State Electron. 21, 1597 (1978).

${ }^{25}$ D. J. Robbins, J. Lumin. 24/25, 137 (1981).

${ }^{26}$ P. J. Dean, D. J. Robbins, S. G. Bishop, J. A. Savage, and P. Porteous, J. Phys. C 14, 2847 (1981).

${ }^{27}$ P. Dahan, V. Fleurov, P. Thurian, R. Heitz, A. Hoffmann, and I. Broser, J. Phys.: Condens. Matter 10, 2007 (1998).

${ }^{28}$ N. Y. Garces, L. Wang, L. Bai, N. C. Giles, L. E. Halliburton, and G. Cantwell, Appl. Phys. Lett. 81, 622 (2002).
${ }^{29}$ T. Monteiro, M. J. Soares, A. Neves, M. Oliveira, E. Rita, U. Wahl, and E. Alves, oral presentation at the First SOXESS European Workshop on ZnO, Autrans, France, 2003 (unpublished).

${ }^{30}$ P. J. Dean, in Inter-Impurity Recombinations in Semiconductors, Progress in Solid State Chemistry Vol. 8, edited by J. O. McCaldin and G. Somorjai (Pergamon, New York, 1973).

${ }^{31}$ A. Y. Polyakov et al., J. Appl. Phys. 94, 400 (2003).

${ }^{32}$ N. Y. Garces, L. Wang, L. Bai, N. C. Giles, L. E. Halliburton, G. Cantwell, and D. B. Eason, J. Appl. Phys. 94, 519 (2003).

${ }^{33}$ T. Monteiro, C. Boemare, M. J. Soares, E. Rita, and E. Alves, J. Appl. Phys. 93, 8995 (2003). 